\title{
Functional Outcomes of Close Pinning and Open Reduction in Pediatric Gartland Type III Supracondylar Fractures: a Comparison
}

\author{
Mohsen Khorrami, Ahmad Dashtbozorg, \\ Mohammadali Ghassemi, Amir Khorrami, \\ Mehdi Ansari* \\ Department of Orthopaedics and Traumatology, Jundishapour \\ University of Medical Sciences, Ahvaz, Iran \\ Study Area: Ahvaz, Iran \\ Coordinates: $31^{\circ} 19^{\prime} 13^{\prime \prime} \mathrm{N} ; 48^{\circ} 40^{\prime} 09^{\prime \prime E}$
}

Key words: Humerus, Children, Gold standard treatment

Project approved from University of Medical Sciences, Ahvaz, Iran. Ethics code: IR.AJUMS.REC.1396.763

\section{Introduction:}

Worldwide supracondylar fractures of the humerus are the most common type of elbow fractures happened in the pediatric age group (Flynn et al., 2014). As per to the modified Gartland classification system the fractures based on extension type are classif ied into 3 types. Type-I is non-displaced, type-II is displaced with an intact posterior cortex, and type-III as the most severe type is completely displaced without cortical contact. These fractures, if left untreated or even treated insignif icantly, might result into severe functional and cosmetic complications including neurovascular injuries, Volkmann's ischemic contracture, valgus deformity, myositis ossificans and limitations in joint motion (Lee \& Kim, 2007; Wingfield et al., 2016). The primary objective in any therapeutic approach for supracondylar humerus fractures must be the complete recovery of the elbow joint motion of the elbow joint and normal cosmetic appearance. For the Gartland type-III fracture of the humerus, the surgical fixation is commonly recommended to prevent mal-union. The standard treatment for Gartland type-III supracondylar fractures is closed reduction and percutaneous pin fixation (Lee \& Kim, 2007). However, an open reduction may be necessary in cases with irreducible fragments, open fractures, as well as in the cases of neurovascular injury (Wingfield et al., 2016). The present study aims for a comparative investigation of the clinical and functional outcomes of
Abstract

A retrospective study in which the medical records of 44 patients of below than 12 years of age with extension-type supracondylar elbow fractures within a time lag of one year were reviewed. Total of 21 patients were treated with closed reduction and percutaneous pinning and 23 had open reduction with pin fixation. According to Flynn's criteria, the outcomes of the open and closed reduction groups did not reveal any significant difference. Although the outcomes of closed reduction showed no superiority over open reduction, it should be the first choice of treatment due to its low morbidity and short hospital stay. Percutaneous pinning is the gold standard treatment in the pediatric Gartland type III humerus fracture, however, in cases with clear indications, a surgeon should not hesitate to apply the open reduction procedure which results in an expected functional outcome.

close and open reduction surgical approaches while the treatment of Gartland type-III pediatric supracondylar humerus fractures.

\section{Materials and methods:}

Our study was based on the medical charts of 44 patients of below than 12 years of age who were treated for extensiontype supracondylar elbow fractures in between September 2016 to September 2017 in the orthopaedic departments of the Hospitals affiliated with the Ahvaz Jundishapur University of Medical Sciences, Ahvaz, Iran. Patients with preoperative neurovascular injuries, open fractures, previous ipsilateral elbow fractures, incomplete data, and loss of follow-up were excluded from the study. Only such patients were included in the study who had a regular postoperative follow up for at least 3 months.

And following the same, a total of 44 patients ( 25 boys and 19 girls) matched the criteria. Mean age of patients at the time of surgery was 6.6 years (range: $2-12$ years), and mean follow-up time was 12 months (range: 6-18 months). A closed reduction under general anesthesia and pin fixation were initially attempted for all type III supracondylar humerus fractures. In cases of inadequate reduction, open reduction and pin fixation were applied with either a Lateral, medial, or posterior approach. Four staff physicians did most parts of the procedure, while residents did the rest under their supervision.

Following the procedure, patients were classified into

*Corresponding Author:mehdiir63@gmail.com 
two groups: i) those who were treated with closed reduction and percutaneous pinning of fracture (Group- $\mathrm{A}, \mathrm{n}=21$ ); and ii) patients who were treated with open reduction and $\mathrm{K}$ wire fixation (Group-B, $\mathrm{n}=\mathbf{2 3}$ ). During percutaneous pinning, the surgeon gradually applied traction to the limb with the elbow extended while the assistant applied counter-traction at the axilla. Fluoroscopy was used to conf irm the translation of the distal of the humerus. While gradually flexing the elbow to about $120^{\circ}$, the surgeon applied direct pressure to the olecranon with the thumb to correct any residual posterior tilting. With the elbow flexed, anteroposterior and lateral radiographs were obtained to evaluate the quality of the reduction.

Two or three pins, $2 \mathrm{~mm}$ in diameter, were inserted from lateral to medial. For cross-pinning, a medial incision centered on the medial epicondyle was performed and the ulnar nerve was isolated, then a $2 \mathrm{~mm}$ diameter pin was inserted from medial to lateral. A lateral pin with the same diameter was inserted percutaneously under fluoroscopic guidance starting at the lateral epicondyle. The wound was closed in two planes with a continuous intradermal suture. In a posterior approach, while a patient was in lateral position a midline $5-6 \mathrm{~cm}$ longitudinal incision extending over the elbow and the distal end of the humerus was made. While the ulnar nerve was identified and carefully mobilized, Paratrigeminal approach was used to expose the fracture line. Fixation was made with crossed pins the following reduction, and fracture stability was ensured clinically and radiologically using fluoroscopy (Fig.-1).

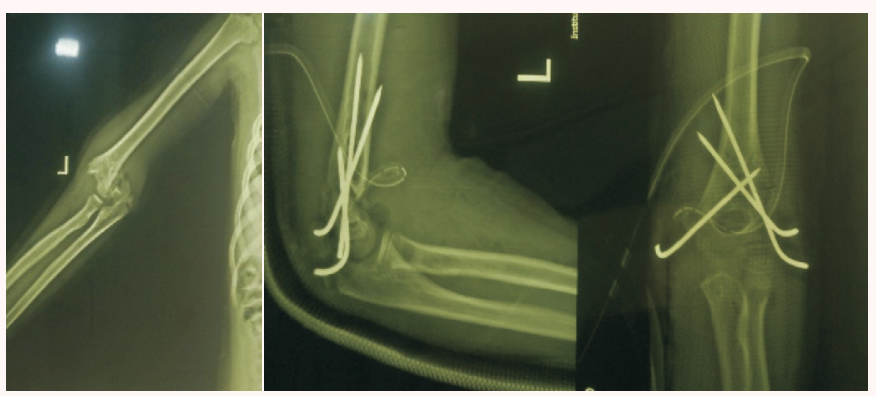

Figure 1: A 9-year-old girl with Gartland type III supracondylar fracture followed by open reduction and crossed pin fixation.

Follow-up and evaluation: in all patients, a long-arm splint were applied for 3 weeks, and range-of-motion (ROM) exercises were started while the pins remained. The pins were removed when sufficient callus was observed radiologically (usually around 6th week postoperatively). All the patients followed a home care ROM exercise program. Children who failed to achieve full ROM 3 month after operation were referred to some physical therapist. All the patients were invited for a final follow-up visit and were evaluated functionally and radiologically. In addition, carrying angle and ROM were measured by goniometry and compared with the contralateral elbow during the final visit. The grading system given by Flynn et al. (2015) was used to assess the functional results of treatment (Table 1 ).
Ambient Science, 2019: Vol. 06h(1); 32-35 DOI:10.21276/ambi.2019.06h.1.oa07

The data were analyzed with the Statistical Package for Social Sciences (SPSS, version 18 for Windows).

Table 1: Flynn et al. (2014)'s criteria followed for the study

\begin{tabular}{lcl}
\hline Outcomes & $\begin{array}{l}\text { Cosmetic factor: } \\
\text { Loss of carrying angle }\end{array}$ & $\begin{array}{l}\text { Functional factor: } \\
\text { Loss of motion }\end{array}$ \\
\hline Satisfactory & & \\
Excellent & $0-5^{\circ}$ & $0-5^{\circ}$ \\
Good & $6-10^{\circ}$ & $6-10^{\circ}$ \\
Fair & $11-15^{\circ}$ & $11-15^{\circ}$ \\
Unsatisfactory & & \\
$\quad$ Poor & $>15^{\circ}$ & $>15^{\circ}$ \\
\hline
\end{tabular}

\section{Results:}

No signif icant difference between the two groups regarding gender, age, and follow-up duration was detected. The mean age in the close pin group was 6.9 years and 6.3 years in the open pin fixation group, so the mean age of the study group was 6.6 years. In this study, the frequency of posteromedial and posterolateral injuries was 70.5\% and $\mathbf{2 9 . 5} \%$, respectively. Postoperative complications like pin tract and wound infection were found in two cases in open pin group which both were superficial (Table-2). Final mean limitation of motion was $2.3 \pm 4.3$ in the close pin group and $2.6 \pm 5.4$ in the open pin group which did not reveal signif icant difference statistically.

Decrease in carrying angle was $1.4 \pm 3.5$ in group A and $2.6 \pm 4.4$ degrees in group B ( $\mathrm{p}>0.33)$. Noticeable clinical limitation of ROM was observed in 5 children (23.8\%) in the close pin group and in 5 children $(21.7 \%)$ in the open pin group.

Table 2: Comparison of the variables between the open and closed reduction surgeries in the participants (patients)

\begin{tabular}{llll}
\hline Parameters & $\begin{array}{l}\text { Close pin } \\
(\mathrm{n}=21)\end{array}$ & $\begin{array}{l}\text { Open pin } \\
(\mathrm{n}=21)\end{array}$ & P value \\
\hline $\begin{array}{llll}\text { Gender } \\
\quad \text { Male }\end{array}$ & 13 & 12 & $<0.55$ \\
$\quad$ Female & 8 & 11 & \\
$\begin{array}{l}\text { Mean age of patients (yrs) } \\
\text { Side affected }\end{array}$ & 6.9 & 6.3 & $<0.46$ \\
$\quad$ Right & 13 & 10 & $<0.24$ \\
$\quad$ Left & 8 & 13 & \\
$\begin{array}{l}\text { Fracture type } \\
\quad \text { Posteromedial }\end{array}$ & 14 & 17 & $<0.74$ \\
$\quad$ posterolateral & 7 & 6 & \\
$\begin{array}{l}\text { Av. follow up (months) } \\
\text { Infection }\end{array}$ & 12.2 & 11.9 & $<0.76$ \\
Revision surgery & 0 & 2 & $<0.48$ \\
\hline
\end{tabular}

Values are given as the number of patients

Loss of ROM was categorized based on the criteria defined by Flynn et al. (2016) which showed 16 (76.1\%) children had excellent scores and $5(23.8 \%)$ patients had good scores in the close pin group. Similarly, in the open pin group, $18(78.2 \%)$ children had excellent scores, 4 (17.3\%) had good scores, and 1 (4.3\%) had a bad score. Cosmetic score, based on the Flynn et al.'s criteria, was excellent in 18 
$(85.7 \%)$ and good in $3(14.2 \%)$ patients in the close pin group. In the open pin group $17(73.9 \%)$, and $6(26.1 \%)$ children had excellent and good scores, respectively (Table 3). Re-operation was required in two patients in the close pingroup compared to one in the open pin group ( $p>0.49$ ).

Table 3: Patients' distribution according to Flynn criteria

\begin{tabular}{lllll}
\hline Parameters & Excellent & Good & Fair & Poor \\
\hline Functional & $0-5$ & $6-10$ & $11-15$ & $? 15$ \\
ROM loss $\left(^{\circ}\right)$ & & & & \\
$\quad$ Close pin & $16(76.1 \%)$ & $5(23.8 \%)$ & - & \\
$\quad$ Open pin & $18(78.2 \%)$ & $4(17.3 \%)$ & & $1(4.3 \%)$ \\
Cosmetic & & & \\
Carrying angle loss $\left(^{\circ}\right)$ & & - \\
\multicolumn{2}{c}{ Close pin } & $18(85.7 \%)$ & $3(14.2 \%)$ & - \\
Open pin & $17(73.9 \%)$ & $6(26.1 \%)$ & \\
\hline
\end{tabular}

Values are given as the number of patients.

\section{Discussion:}

Supracondylar fracture of the humerus is the most common surgically treated fracture during childhood. Treatments for displaced supracondylar humerus fracture are closed reduction and internal fixation or open reduction and internal fixation, and numerous surgical techniques have been described in the literature (Omid et al., 2008). Anatomical reduction and stable fixation with good cosmetic appearance and full ROM are the proper treatment goals (Bombaci et al., 2007) Closed reduction and percutaneous pinning is the commonly accepted primary method for treatment. However, in some circumstances such as soft tissue entrapment, severely displaced fracture, very edematous elbow, open fracture, and neurovascular injury, an open reduction may be required (Kazimoglu et al., 2009).

The range of motion limitation of the elbow after supracondylar fractures can be caused by soft tissue injuries, posttraumatic remodeling, fibrous surgical scars, and mal-union. In our study, the difference between the two groups regarding the loss of ROM was not statistically significant ( $p>0.56)$, and both groups had an excellent or good range of motion. Similar results were shown by Kumar et al. (2016) and Yaokreh et al. (2012). The role of physical therapy in patients with restricted elbow motion is always a controversial issue, as the physical activities which children are naturally inclined to are resultant in some degrees of self-rehabilitation.

Cosmetic satisfaction could be attributed to the absence of residual deformity and location of incisions. Numerous studies reported various functional and cosmetic outcomes with different approach types. Barlas \& Baga (2005) reported 90.69\% good-excellent functional and cosmetic results with a medial approach in 2 years follow-up using the Flynn's criteria. Eren et al. (2005) compared lateral and medial approaches and found goodexcellent results with $95 \%$ functionally and $100 \%$ cosmetically with lateral approach; whereas $100 \%$ both functionally and cosmetically with a medial approach. Bamrungthin (2008) compared lateral and posterior approaches and good-excellent results were found with $80 \%$ in the lateral approach and $80.7 \%$ in the posterior approach. However, for both studies, the difference in outcomes of lateral and medial approaches were not statistically significant.

In our study, most used approaches for open pin group were: posterior (12), medial (6), and lateral in 5 cases. Only five cases in posterior approach group had an unsatisfactory cosmetic appearance with hypertrophic scars, which was not statistically significant.

Eren et al. (2008) conducted a study to evaluate the relationship between the fracture displacement and cubitus varus deformity in displaced supracondylar humerus fractures. They concluded that although anatomic reduction has been achieved by surgical treatment without loss of reduction, there is still a risk for cubitus varus deformity for Type III fractures due to the initial compression of the medial column. Khan et al. (2007) conducted a study on 6o children with Gartland type-III supracondylar humerus fracture with only one patient developed cubitus varus deformity. In our study, we did not encounter any case of cubitus varus deformity or iatrogenic ulnar nerve injury. However, the limitations of our study were retrospective design, small sample size, absence of randomization, short term follow up, and not having the same rehabilitation center.

Conclusively, percutaneous pinning is the gold standard treatment in the pediatric Gartland type III humerus fracture. However, in cases with clear indications, a surgeon should not hesitate to apply the open reduction procedure that would result in more desired functional outcome..

\section{Acknowledgment:}

We are thankful to particinats and the other medical staffs who have helped us to conclude this study.

\section{References:}

Barlas, K. \& Baga, T. (2005): Medial approach for fixation of displaced supracondylar fractures of the humerus in children. Acta Orthop. Belg., 71(2):149-153.

Bamrungthin, N. (2008): Comparison of posterior and lateral surgical approach in management of type III supracondylar fractures of the humerus among the children. J. Med. Assoc. Thai., 91(4):502-506.

Bombaci, H., Gereli, A., Kucukyazici, O., Gorgec, M., \& Deniz, G.(2007): The effect of surgical exposure on the clinic outcomes of supracondylar humerus fractures in children. Ulus. Travma Acil Cerrahi Derg., 13(1):49-54.

Eren, A., Guven, M., Erol, B., Akman, B. \& Ozkan, K (2008): Correlation between posteromedial or posterolateral displacement and cubitus varus deformity in supracondylar humerus fractures in children. L. Child Orthop., 2(2):85-89.

Eren, A., Ozkut, A.T., Altintas, F., \& Guven, M.(2005): Comparison 


\section{ORIGINAL ARTICLE}

between the lateral and medial approaches in terms of functional and cosmetic results in the surgical treatment of type III supracondylar humeral fractures in children. Acta Orthop. Traumatol. Turc., 39(3):199-204.

Flynn, J.M., Skaggs, D.L. \& Waters, P.M. (2014): Rockwood and Wilkins'. Fractures in Children, 8th ed. Pub. by: Lippincott Williams and Wilkins. 1252 pp.

Kazimoglu, C., Cetin, N., Sener, M., Agus, H., \& Kalanderer, O. (2009): Operative management of type III extension supracondylar fractures in children. Int. Orthop., 33(4):10891094.

Khan, A.Q., Goel, S., Abbas, M. \& Sherwani, M.K.(2007): Percutaneous K-wiring for Gartland type III supracondylar humerus fractures in children. Saudi Med. J., 28(4):603-6o6.

Lee, H.Y. \& Kim, S.J. (2007): Treatment of displaced supracondylar fractures of the humerus in children by a pin leverage technique. J. Bone Joint Surg. Br., 89(5):646-650.

Omid, R., Choi, P.D., \& Skaggs, D.L. (2008): Supracondylar humeral fractures in children. J. Bone Joint Surg. Am., 9o(5):1121-1132.
Ambient Science, 2019: Vol. 06h(1); 32-35 DOI:10.21276/ambi.2019.06h.1.oa07

Prashant, K., Lakhotia, D., Bhattacharya, D.T., Mahanta, K.A. \& Ravoof, A. (2016): A comparative study of two percutaneous pinning techniques (lateral vs medial-lateral) for Gartland type III pediatric supracondylar fracture of the humerus. $J$. Orthop. Traumatol., 17(3):223-229.

Turkmen, F., Toker, S., Kesik, K., Korucu, I.H. \& Acar, M.A. (2016): Comparison of lateral versus triceps-splitting posterior approach in the surgical treatment of pediatric supracondylar humerus fractures. Ulus. Travma Acil Cerrahi Derg., 22(5):483.

Wingfield, J.J., HO, C.A., Abzug, J., Ritzman, T.F. \& Brighton, B. (2016): Open Reduction Techniques for Supracondylar Humerus Fractures in Children. Instr. Course Lect., 65: 361369.

Yaokreh, J.B., Gicguel, P., Schneider, L., Stanchina, C., Karger, C., Saliba, E., Ossenou, O. \& Clavert, J.M. (2012): Compared outcomes after percutaneous pinning versus open reduction in paediatric supracondylar elbow fractures. Orthop. Traumatol. Surg. Res., 98(6): 645-651. 